\title{
Dact1 Is a Postsynaptic Protein Required for Dendrite, Spine, and Excitatory Synapse Development in the Mouse Forebrain
}

\author{
Nathan D. Okerlund, ${ }^{4}$ Saul Kivimäe, ${ }^{1}$ Cheuk Ka Tong, ${ }^{4}$ I-Feng Peng, ${ }^{3}$ Erik M. Ullian, ${ }^{2,3,4}$ and Benjamin N. R. Cheyette ${ }^{1,4}$ \\ Departments of ${ }^{1}$ Psychiatry, ${ }^{2}$ Physiology, and ${ }^{3}$ Ophthalmology and ${ }^{4}$ Neuroscience Graduate Program, University of California, San Francisco, San \\ Francisco, California 94158
}

\begin{abstract}
Dact1 (Dapper/Frodo), an intracellular phosphoprotein that binds Dishevelled, catenins, and other signaling proteins, is expressed in the developing and mature mammalian CNS, but its function there is unknown. Dact1 colocalized with synaptic markers and partitioned to postsynaptic fractions from cultured mouse forebrain neurons. Hippocampal neurons from Dact1 knock-out mice had simpler dendritic arbors and fewer spines than hippocampal neurons from wild-type littermates. This correlated with reductions in excitatory synapses and miniature EPSCs, whereas inhibitory synapses were not affected. Loss of Dact1 resulted in a decrease in activated Rac, and recombinant expression of either Dact1 or constitutively active Rac, but not Rho or Cdc42, rescued dendrite and spine phenotypes in Dact1 mutant neurons. Our findings suggest that, during neuronal differentiation, Dactl plays a critical role in a molecular pathway promoting Rac activity underlying the elaboration of dendrites and the establishment of spines and excitatory synapses.
\end{abstract}

\section{Introduction}

Dendrite, dendritic spine, and synapse development rely on overlapping biochemical pathways (Kayser et al., 2008; Sala et al., 2008; Urbanska et al., 2008). Dendrite and spine development require neurite extension and remodeling through extensive elaboration and alteration of the cytoskeleton; synapse development similarly requires specialized cytoskeletal refinements. Dendrite development involves adhesive interactions with neighboring cells and the extracellular milieu; spine and synapse development similarly require specialized cell adhesion and signaling, with coordinated organization of presynaptic structures in the axon and postsynaptic structures in the adjacent dendrite. Molecular mechanisms underlying dendrite, spine, and synapse development are likely to contribute to the maintenance and plasticity of these structures later in life (Dalva et al., 2007).

Dact (Dapper antagonist of catenin; Dapper/Frodo) genes encode a small family of vertebrate intracellular phosphoproteins that regulate signaling through binding to both cytoplasmic and nuclear partners. Family members are similar in size (600-850 $\mathrm{aa} / 100-120 \mathrm{kDa}$ ) and are distinguished by a leucine zipper motif near the $\mathrm{N}$ terminus and a PDZ-binding (postsynaptic density

\footnotetext{
Received Jan. 21, 2010; accepted Feb. 18, 2010.

This work was supported by a National Science Foundation Graduate Research Fellowship (N.D.O.), Autism Speaks Predoctoral Fellowship 4654 (N.D.O.), a National Alliance for Research on Schizophrenia and Depression Young Investigator Award (S.K.), a Simons Foundation Autism Research Initiative (B.N.R.C., E.M.U.), and National Institutes of Health Grant R21MH085995 (B.N.R.C.). We thank our neighbors Louis Reichardt, John Rubenstein, Holly Ingraham, and all members of our laboratory and scientific neighborhood. Confocal microscopy was performed at the Nikon Imaging Center (California Institute for Quantitative Biosciences, University of California, San Francisco, San Francisco, (A) with advice from $K$. Thorn.

Correspondence should be addressed to Benjamin Cheyette, University of California, San Francisco, MC 2611, Rock Hall Room 284D, 1550 4th Street, San Francisco, CA 94158-2324. E-mail: bc@ucsf.edu.

DOI:10.1523/JNEUROSCI.0354-10.2010

Copyright $\odot 2010$ the authors $\quad 0270-6474 / 10 / 304362-07 \$ 15.00 / 0$
}

95/Discs large/zona occludens-1-binding) motif at the C terminus, each embedded within larger conserved domains (Cheyette et al., 2002; Fisher et al., 2006). In addition to modulating Wnt/ $\beta$-catenin signaling through direct interactions with Dishevelled proteins (Cheyette et al., 2002; Gloy et al., 2002), Dact1 has been proposed to bind and stabilize p120-catenin, thereby promoting $\beta$-catenin-independent signaling to the nucleus (Park et al., 2006), to regulate transcription through direct binding to a subclass of the LEF/TCF (lymphoid enhancing factor/T cell factor) family of DNA-binding proteins and to histone deacetylase (Hikasa and Sokol, 2004; Gao et al., 2008), to functionally interact with the cell division cycle kinase regulatory protein Dbf4 (Brott and Sokol, 2005), and to bind and regulate levels of the planar cell polarity transmembrane protein Vangl2 (Suriben et al., 2009).

All three mammalian members of the Dact family (Dact1, Dact2, and Dact3) are expressed in the nervous system (Fisher et al., 2006), and Dact1 in particular shows intriguingly regulated and bilaterally asymmetric expression patterns in the developing telencephalon (Sun et al., 2005; Long et al., 2009), leading us to explore its function in differentiating neurons. Given the preponderance of PDZ-binding interactions in developing dendritic spines and synapses (Dalva et al., 2007), we were particularly interested in exploring roles for Dact1 in these neurodevelopmental events. Using custom-generated recombinant human monoclonal antibodies, confocal microscopy, and subcellular fractionation techniques, we found that Dactl colocalized with synaptic markers and partitioned into postsynaptic fractions. Taking advantage of gene targeted mice, we investigated requirements for Dact1 in dendrite and synapse development. Cultured hippocampal neurons (HCNs) derived from newborn Dact $^{-/-}$ mice (mutants) had simpler dendritic arbors, fewer dendritic spines, and fewer excitatory synapses than HCNs from wild-type litter- 

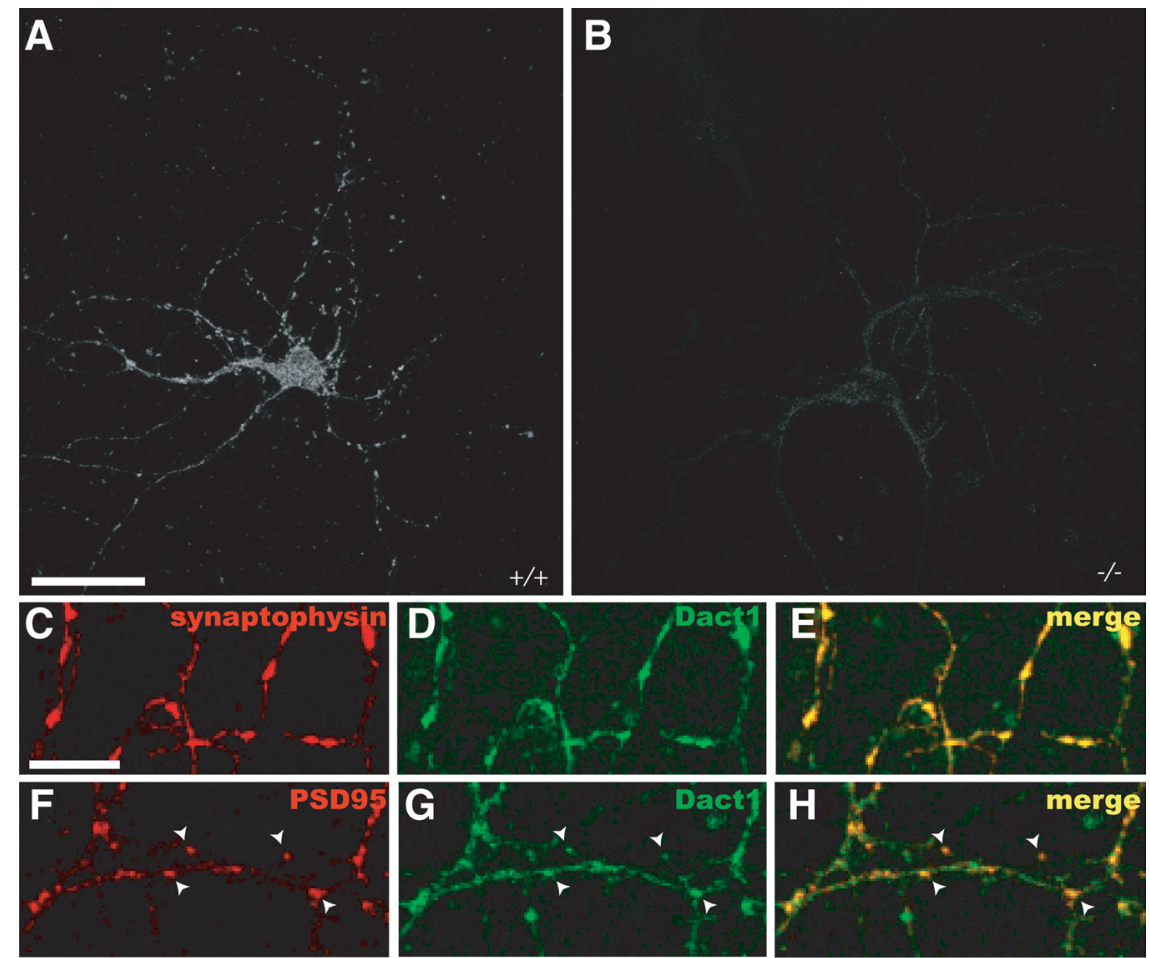

I

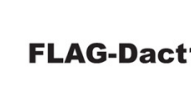

$\mathbf{H}$

P1

$\mathbf{s 2}$

P2'

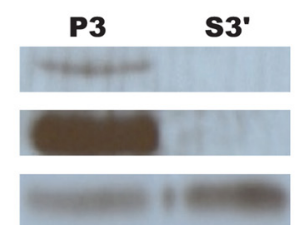

synaptophysin

Figure 1. Dact1 is present at forebrain synapses. $\boldsymbol{A}-\boldsymbol{H}$, Immunocytochemistry, HCNs. $\boldsymbol{A}, \boldsymbol{B}$, Anti-Dact1 in wild type $(\boldsymbol{A})$ and Dact1 $^{-/-}(\boldsymbol{B}) . \mathbf{C}-\boldsymbol{H}$, Confocal microscopy. $\boldsymbol{C}-\boldsymbol{E}$, Anti-Dact1 versus synaptophysin. $\boldsymbol{F}-\boldsymbol{H}$, Anti-Dact1 versus PSD95; arrowheads indicate postsynaptic sites. I, FLAG-Dact1 expressed in forebrain cortical neurons is enriched in postsynaptic fractions. H, Homogenate; $\mathrm{P1}$, crude pellet; $\mathrm{S2}$, supernatant; $\mathrm{P2}{ }^{\prime}$, crude synaptosomes; $\mathrm{P3}, \mathrm{S3}{ }^{\prime}$, postsynaptic density and presynaptic fractions, respectively. FLAG-Dact1 partitions with PSD95, including in P3 but not S3'. Scale bars: $\mathbf{A}, \mathbf{B}, 30 \mu \mathrm{m} ; \mathbf{C}-\boldsymbol{H}, 10 \mu \mathrm{m}$.

mates. Golgi-Cox staining revealed similarly reduced dendritic spines in hippocampal pyramidal neurons of mature mice specifically lacking Dact1 in forebrain glutamatergic neurons (conditional mutants). Recombinant expression of Dact1 in cultured Dact $^{-/-}$HCNs partially restored dendrite complexity and fully rescued spine numbers but induced morphological distortions consistent with hyperactivation of the small GTPase Rac. Concordantly, loss of Dact1 led to significant decrements in activated Rac, as measured in hippocampal lysates from conditional mutants. Conversely, recombinant expression of constitutively active Rac, but not other Rho family GTPases, rescued dendrite and spine phenotypes in Dact $^{-/-}$HCNs. Our findings suggest that, within differentiating mammalian forebrain neurons, Dactl contributes to dendrite, spine, and excitatory synapse formation via a molecular pathway that promotes Rac activity.

\section{Materials and Methods}

Recombinant DNA. Rac, Rho, and Cdc42 cDNAs in pCDNA3.1 (Invitrogen) were obtained from the cDNA Resource Center. Phosphorylated enhanced green fluorescent protein (EGFP)-C1 (Clontech) was cotransfected, except for the Dactl cDNA, in which a pCAGGS vector with an internal ribosomal entry site 2-EGFP was used (Niwa et al., 1991; Fisher et al., 2006).

Primary culture. The primary culture was as described by Elia et al. (2006).

Dact1 antibody. Recombinant human monoclonal antibodies were obtained by screening a synthetic naive library (Rothe et al., 2008) for recom- binant human $\mathrm{F}\left(\mathrm{ab}^{\prime}\right)$ fragments recognizing a bacterially expressed and purified glutathione $S$-transferase (GST) fusion corresponding to the $\mathrm{N}$ terminus of mouse Dactl (amino acids 1-328). Antigen was immobilized on a MaxiSorp microtiter plate, and three selection rounds were performed. To eliminate panDact family antibodies, the library was blocked with excess $(25 \mu \mathrm{g} / \mathrm{ml})$ GST fusion proteins corresponding to mouse Dact2 (amino acids 146-273) and mouse Dact3 (amino acids 175-308). Two clones, AbD06365 and AbD06366 (AbD Serotec), gave indistinguishable staining patterns in HCNs but corresponded to separate epitopes (XiaoYong Yang and Benjamin N. R. Cheyette, unpublished observation). Figure $1, A$ and $B$, was obtained using AbD06365 in a bivalent miniantibody format containing a Myc and a $6 \times$ His tag, whereas Figure $1 C-H$ was obtained using AbD06366 in the same format.

Immunocytochemistry. After fixation, cells were blocked for $1 \mathrm{~h}$ at room temperature (blocking medium: 10\% goat serum in PBS), and then primary antibody was added: rabbit anti-synaptophysin (1:200; Zymed), mouse anti-postsynaptic density-95 (PSD95) (1:200; Thermo Fisher Scientific), rabbit anti-vesicular glutamate transporter 1 (VGlut1), rabbit antivesicular GABA transporter (VGAT), and mouse anti-gephyrin (all 1:200; Synaptic Systems). After three 5 min washes in PBT (PBS plus $0.1 \%$ Triton $\mathrm{X}-100$ ), fluorescent secondary antibodies (1:200; Alexa 405, Alexa 488, or Alexa 568 anti-rabbit or anti-mouse antibodies; Invitrogen) were applied in blocking medium for $1 \mathrm{~h}$ at room temperature. After three 5 min washes (PBT), cells were washed with deionized water and mounted in Mowiol (Thermo Fisher Scientific). For Dact1, primary antibodies were applied at $5 \mu \mathrm{g} / \mathrm{ml}$ and then visualized with a goat anti-human $\mathrm{F}\left(\mathrm{ab}^{\prime}\right) 2$ conjugated to fluorescein or Texas Red (1:200) (Jackson ImmunoResearch) or with biotinylated anti-His antibody (1: 200; AbD Serotec), followed by Texas Red-avidin (1:60; Vector Laboratories). Polymerized filamentous actin (F-actin) was visualized using rhodamine-conjugated phalloidin (1:1000; Invitrogen) in PBS for $1 \mathrm{~h}$ at room temperature, followed by three $5 \mathrm{~min}$ washes (PBT), one wash $\left(\mathrm{H}_{2} \mathrm{O}\right)$, and then mounted in Mowiol (Thermo Fisher Scientific).

Visualization and quantitation. Cells were visualized on Nikon CS1i upright spectral or A1 upright confocal microscopes, and images were analyzed with NIH ImageJ software and Sholl analysis plugin (Anirvan Ghosh, University of California, San Diego, La Jolla, CA). Dendritic projections were binned on the basis of their morphology as filopodia, thin spines, mushroom spines, or stubs as described previously (Hering and Sheng, 2001).

Electrophysiology. Electrophysiology was as described by Lee et al. (2008).

Subcellular fractionation. Cortical neuronal cultures were prepared as described previously (Cobos et al., 2007), transfected ( $10 \mathrm{~d}$ in vitro) with FLAG-tagged Dact1, and homogenized (14 d in vitro). Crude synaptosomes were prepared by serial centrifugation (Hell and Jahn, 1994) and separated into presynaptic and postsynaptic fractions by Triton X-100 detergent extraction and centrifugation as described previously (Garside et al., 2009).

Immunoblotting. Immunoblotting was performed as described previously (Suriben et al., 2009) with the following: mouse $\alpha$-, $\beta$-, p120-, and $\delta$-catenin antibodies (1:200; BD Biosciences); rabbit anti- $\beta$-actin (1:1000; Santa Cruz Biotechnology); rabbit anti-synaptophysin (Zymed); mouse anti-PSD95 (NeuroMAB, University of California, Davis, Davis, CA); and mouse antiFLAG (Sigma-Aldrich) (1:1500); and HRP-conjugated secondary antibodies (1:8000; Thermo Fisher Scientific). 
Quantitative reverse transcription-PCR. HCNs were lysed with Trizol, and mRNA was isolated according to the instructions of the manufacturer (Invitrogen). Equal amounts of mRNA per sample were processed side by side: cDNA was prepared and quantitative reverse transcription (RT)-PCR was performed as described previously (Fisher et al., 2006) using primers for Fos, Fosl, (Abe and Takeichi, 2007), Axin1 (Dao et al., 2007), and Axin2 (Suriben et al., 2009).

Rac activity. Hippocampi were dissected from adult mice, homogenized, and analyzed for GTP-bound activated Rac versus total Rac by GST- $4 \beta$-phorbol 12,13-dibutyrate (PDB) affinity precipitation as described previously (Habas and He, 2006).

Genetics. All Dact1 alleles were derived from Dact1 ${ }^{\text {tm1.1Bnrc }}$ (Suriben et al., 2009). Cultured HCNs were from littermate offspring $\left(\right.$ Dact $1^{+/+}$, Dactl $^{+/-}$, and Dact1 ${ }^{-/-}$) of a Dact1 ${ }^{+/-}$intercross. No significant phenotypic differences were observed between Dact $1^{+/+}$and Dact1 ${ }^{+/-}$ HCNs (data not shown); unless otherwise indicated, all comparisons are Dact1 ${ }^{-/-}$(mutant) versus Dact $^{+/+}$(wild type). Adult mice lacking Dactl selectively in cortical and hippocampal glutamatergic neurons were created through genetic combinations of the neo ${ }^{-}$Dact $1^{f l o x}$ allele and transgenic Cre driven by the Emxl promoter $\left(E m x 1^{t m 1(c r e) K r j}\right)$ (Gorski et al., 2002). For Golgi-Cox, the parental cross was Dact1 ${ }^{+/-}$; $E m x 1^{\text {Tml } 1(c r e) K r j /+} \times$ Dact $^{\text {flox } / f l o x}$; experimental progeny were Dact ${ }^{\text {flox/- }}$;Emx $1^{\text {Tml(cre)Krj/+ }}$ (con-

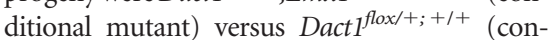
trol). For Rac activity, the parental cross was Dact $^{\text {flox/+ }} ; E m x 1^{\text {Tml (cre)Krj/+ }} \times$ Dact $1^{\text {flox/flox }}$; experimental progeny were Dact $1^{\text {flox/flox; }}$; Emxl $1^{\text {Tml } 1 \text { (cre)Krj/+ }}$ (conditional mutant) versus Dact $^{\text {flox/flox; }^{+/+}}$or Dact1 flox/+; +/+ $^{\text {(control). }}$

Golgi-Cox staining. Golgi-Cox staining was performed according to the instructions of the manufacturer (FD GolgiStain kit; FD NeuroTechnologies).

Statistics. All $p$ values were calculated by unpaired parametric two-tailed $t$ test (two comparisons) or one-way ANOVA (three or more comparisons) using Prism software (GraphPad Software).

\section{Results}

HCNs stained with Dactl-specific anti-

bodies displayed a punctate signal distribution in somata and along neurites (Fig. 1A). This staining, which was absent from Dact $^{-/}$HCNs (Fig. 1B), colocalized with synaptic markers, including at punctate intensities of the excitatory postsynaptic marker protein PSD95 likely to correspond to postsynaptic sites (Fig. $1 C-H)$. Supporting a postsynaptic localization for Dact1, immunoblotting of subcellular fractions derived from forebrain cortical cultures revealed that recombinantly expressed FLAG-Dact1 copartitioned with PSD95 in postsynaptic, as opposed to presynaptic, fractions (Fig. 1I).

By visual inspection, Dact1 mutant HCNs in culture had simpler dendritic arbors and fewer dendritic spines than HCNs from wild-type littermates (Fig. $2 A, B$ ). Spine density was rescued and arbor complexity was partially rescued by recombinant expression of Dact1 in mutant neurons (Fig. 2C). Quantitation using
Sholl analysis showed that mutant HCNs had reductions in dendrite branch crossings at all distances from the soma (Fig. 2D). Dendritic spines were also affected (Fig. 2, compare $A^{\prime}, B^{\prime}$ ): the number of spines per $10 \mu \mathrm{m}$ of dendrite length was highly significantly reduced in mutant HCNs $\left(\right.$ Dact $1^{+/+}, 2.79 \pm 0.13$ vs Dact $^{-1-}, 2.00 \pm 0.15, p=0.0021$ ) (Fig. 2E). In addition to a decrease in spine number, mutant HCNs showed a significant increase in the proportion of immature filopodial "spines" relative to mature spines (thin spines, mushroom spines, and stubs) $\left(\right.$ Dact $^{+/+}, 0.13 \pm 0.02$ vs Dact $\left.1^{-/-}, 0.35 \pm 0.05, p=0.0036\right)$ (Fig. 2F). Mutant HCNs recombinantly expressing Dact1 (Dact1-rescued) produced a large number of short dendrites (Fig. 2C,D); interestingly, the density of dendritic spines and proportion of mature spines were both restored to wild-type levels in Dact1-rescued HCNs (spines per $10 \mu \mathrm{m}$ in wild type, $2.79 \pm 0.13$ vs Dact1-rescued, $2.83 \pm 0.15, p=0.86$; filopodia/total spines 

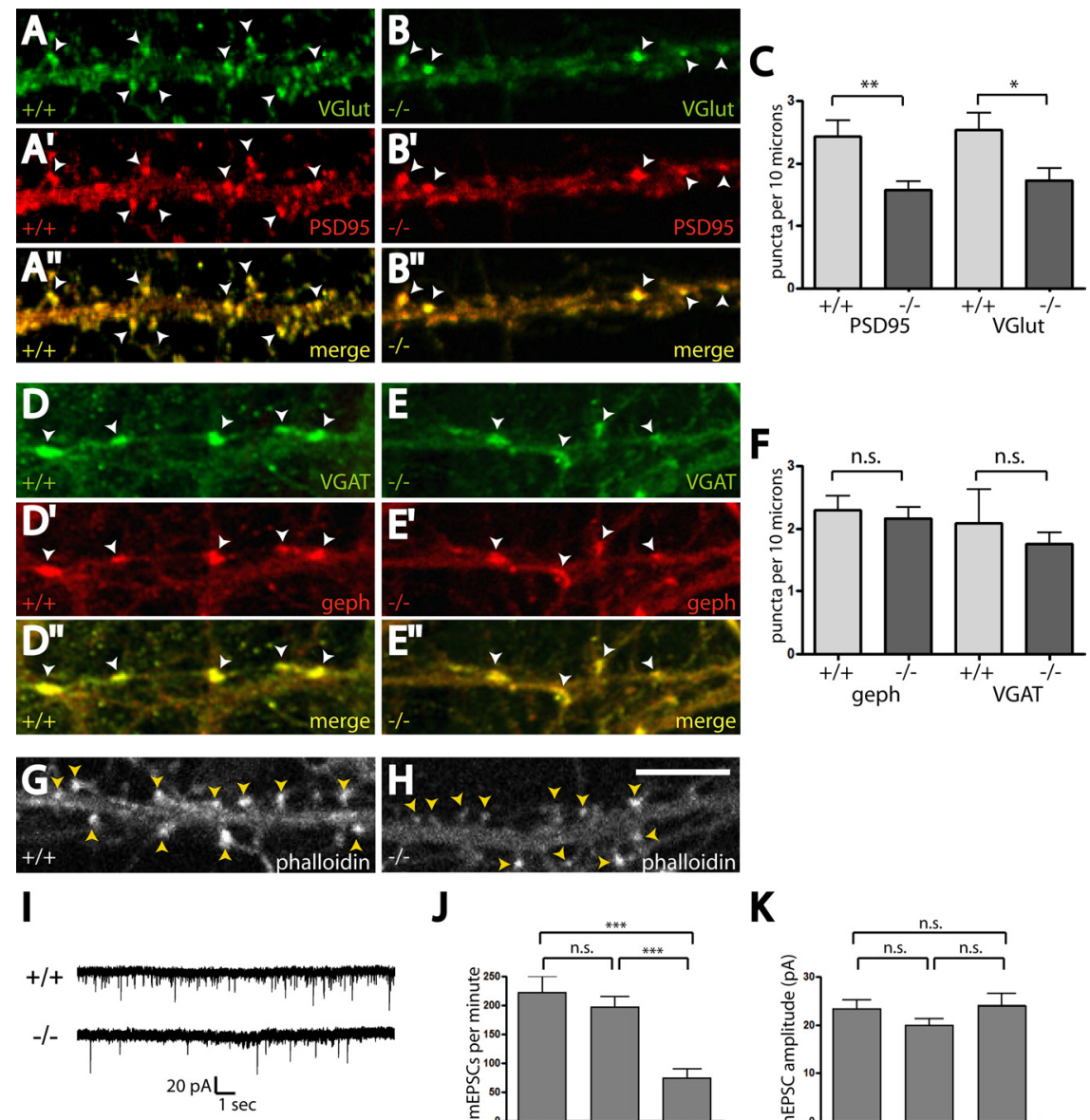

J

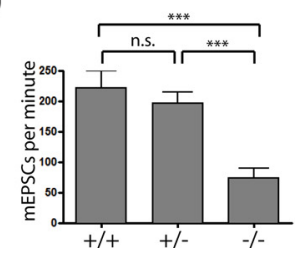

\section{K}

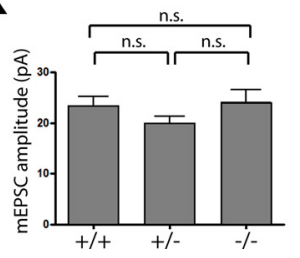

Figure 3. Dact1 mutant neurons have fewer excitatory synapses; inhibitory synapses are spared. $\boldsymbol{A}-\boldsymbol{I}$, Immunocytochemistry, dendrites. $\boldsymbol{A}, \boldsymbol{B}$, VGlut1: $\boldsymbol{A}$, wild type; $\boldsymbol{B}$, Dact $1^{-1-} . \boldsymbol{A}^{\prime}, \boldsymbol{B}^{\prime}$, PSD95: $\boldsymbol{A}^{\prime}$, wild type; $\boldsymbol{B}^{\prime}$, Dact $1^{-/-} . \boldsymbol{A}^{\prime \prime}, \boldsymbol{B}^{\prime \prime}$, Colocalization. There are fewer excitatory synapse puncta (arrowheads) on mutant neurites. C, Quantitation. $\boldsymbol{D}, \boldsymbol{E}$, VGAT: $\boldsymbol{D}$, wild type; $\boldsymbol{E}$, Dact $1^{-1-}$. $\boldsymbol{D}^{\prime}, \boldsymbol{E}^{\prime}$, Gephyrin: $\boldsymbol{D}^{\prime}$, wild type; $\boldsymbol{E}^{\prime}$, Dact1 $1^{-/-} . \boldsymbol{D}^{\prime \prime}, \boldsymbol{E}^{\prime \prime}$, Colocalization. Inhibitory synapse puncta (arrowheads) appear no different on mutant neurites. $\boldsymbol{F}$, Quantitation. $\mathbf{G}, \boldsymbol{H}$, Phalloidin (actin). Compared with wild type (G), the intensity, size, and number of puncta (arrowheads) are diminished in Dact $1^{-/-}$neurites $(\boldsymbol{H})$. $\boldsymbol{I}-\boldsymbol{K}$, Electrophysiology. $\boldsymbol{I}$, Representative trace of mEPSCs in wild type (top) versus Dact $1^{-/-}$(bottom). J, mEPSC numbers are reduced in Dact $1^{-/-}$relative to controls. $\boldsymbol{K}, \mathrm{mEPSC}$ amplitude is unaffected. Scale bar, $10 \mu \mathrm{m} .{ }^{*} p<0.05,{ }^{* *} p<0.01,{ }^{* * *} p<0.001$; n.s., Not significant.

in Dact $^{+/+}, 0.13 \pm 0.03$ vs Dact1-rescued, $0.24 \pm 0.04, p=$ 0.085 ) (Fig. 2 $C^{\prime}, E, F$ ). To summarize, cultured HCNs lacking Dactl displayed significant decreases in dendrite complexity, spine numbers, and morphological indices of spine maturation; phenotypes that were all rescued or partially rescued by recombinant restoration of Dact1.

Mice with constitutive loss of Dact1 generally die within a day of birth as a result of complex urogenital and gastrointestinal malformations (Suriben et al., 2009). To test whether phenotypic alterations observed in dissociated HCNs correspond to crucial functions of Dact1 in the mammalian forebrain, we took advantage of Cre-loxP technology to selectively eliminate Dact1 from glutamatergic neurons in the cerebral cortex and hippocampus during development (Gorski et al., 2002). As assessed by Nissl stain, the cellular organization and regional anatomy of brains from such conditional mutant mice was indistinguishable from wild type (data not shown). To test the hypothesis that loss of Dact1 has specific developmental consequences on neurite complexity in these animals, we performed Golgi-Cox staining on brains taken from adult (9-10 week old) mice, analyzing pyramidal neurons in the CA1 region of the hippocampus. CA1 hippocampal neurons in conditional mutant brains showed a significant decrease in spine density per $10 \mu \mathrm{m}$ on apical dendrites (control, $2.0 \pm 0.4$ vs conditional mutant, $1.0 \pm 0.2, p=0.014)$, as well as on secondary dendrites (control, $4.1 \pm 0.2$ vs conditional mutant, $2.6 \pm 0.2, p=0.0044$ ) (Fig. $2 G-J)$. Neurons in conditional mutant brains also showed highly significant decreases in spine length (control, $3.2 \pm 0.2$ $\mu \mathrm{m}$ vs conditional mutant, $1.62 \pm 0.06$ $\mu \mathrm{m}, p<0.0001)$ and in spine head width (control, $1.8 \pm 0.1 \mu \mathrm{m}$ vs conditional mutant, $1.14 \pm 0.03 \mu \mathrm{m}, p<0.0001)$. Together with the data from dissociated HCNs, these histological data demonstrate that Dactl plays a critical role during dendrite and spine development in the mouse hippocampus.

Excitatory synapses in mutant HCNs as assessed by puncta visualized with antibodies specific for VGlut1 (an excitatory presynaptic marker) and PSD95 (an excitatory postsynaptic marker) were significantly reduced when compared with those in HCNs derived from wild-type littermates (puncta per $10 \mu \mathrm{m}$ in Dact ${ }^{+/+}$, $2.4 \pm 0.3$ vs Dact $^{-/-}, 1.57 \pm 0.14, p<$ 0.0073) (Fig. 3A-C). However, inhibitory synapses, as assessed by puncta visualized with antibodies specific for VGAT (presynaptic inhibitory marker) and gephyrin (postsynaptic inhibitory marker), were unchanged in mutant HCNs (puncta per $10 \mu \mathrm{m}$ in Dact $1^{+/+}, 2.3 \pm 0.2$ vs Dact $^{-/-}, 2.2 \pm 0.2, p=0.67$ ) (Fig. 3D$F)$. Consistent with the loss of mature spines and associated excitatory synapses, F-actin puncta, found in mature spine heads, were reduced in both intensity and number in mutant $\mathrm{HCN}$ s relative to wild type (puncta per $10 \mu \mathrm{m}$ in $\operatorname{Dact}^{+/+}$, $3.5 \pm 0.2$ vs Dact $1^{-/-}, 2.56 \pm 0.15, p=$ 0.0005) (Fig. 3G,H). Electrophysiological analysis of mutant HCNs confirmed a deficit in excitatory synapses in these neurons (Fig. 3I). The frequency of miniature EPSCs (mEPSCs) was highly significantly reduced in mutant $\mathrm{HCN}$ compared with either wild-type or heterozygous HCNs (mEPSCs per minute in Dact $^{+/+}, 220 \pm 30$ vs Dact1 ${ }^{+/-}, 200 \pm 20$ vs Dact $1^{-/-}, 75 \pm 15$, $p<0.001$ ) (Fig. 3J), but the amplitude of mEPSCs was normal $\left(\right.$ Dact $^{+/+}, 23 \pm 2 \mathrm{pA}$ vs Dact $1^{+/-}, 20 \pm 1 \mathrm{pA}$ vs Dact $^{-/-}, 24 \pm$ $2 \mathrm{pA}, p=0.26)$ (Fig. $3 K)$. In summary, the molecular marker and electrophysiological data demonstrated a marked reduction in numbers of excitatory synapses in mutant HCNs, whereas inhibitory synapses were spared.

There is evidence that Dactl homologs can form complexes with and regulate levels of catenin proteins, particularly p120catenin, which has established roles at synapses (Elia et al., 2006; Park et al., 2006). We accordingly examined levels of p120catenin (Ctnnd1) and the related $\delta$-catenin (Ctnnd2) protein in mutant HCNs. Western blot revealed no detectable differences in levels of these $\delta$-catenin family members, nor in total levels of either the $\alpha$-catenin or $\beta$-catenin proteins in mutant compared with wild-type HCNs (Fig. 4A). This was surprising because in other contexts Dact proteins have been proposed to modulate the 
A
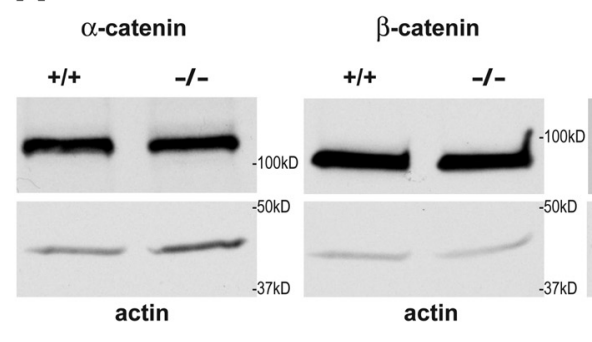

$\delta$-catenin

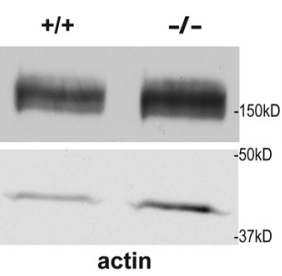

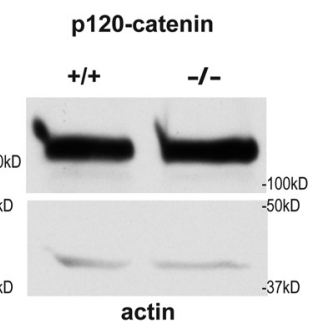

actin

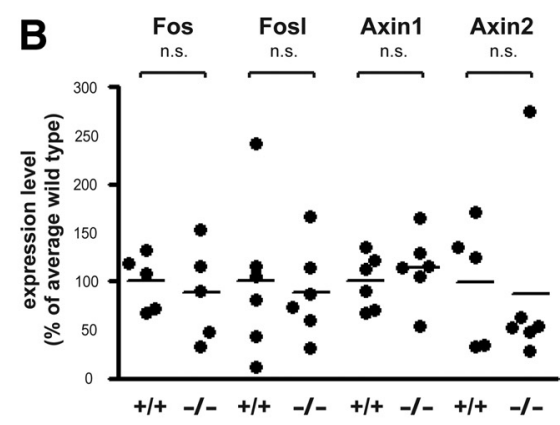

C

D
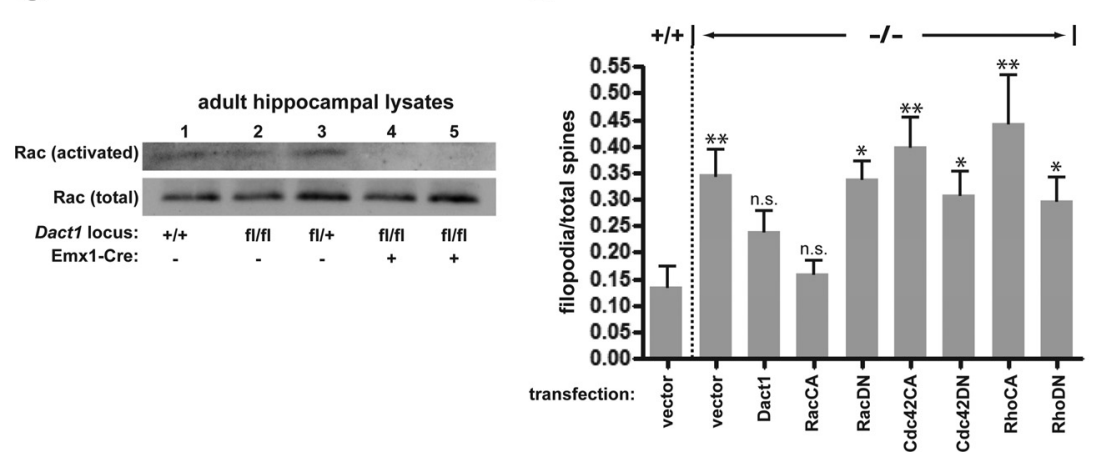

E

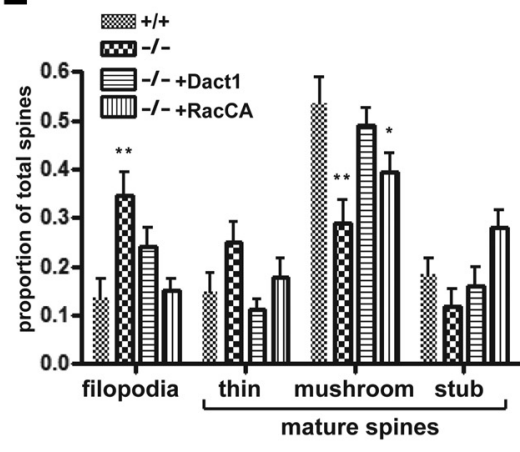

Figure 4. Dact1 mutant neural phenotypes reflect hypoactive Rac. $\boldsymbol{A}, \boldsymbol{B}, \boldsymbol{D}, \boldsymbol{E}$, Cultured HCNs; $\boldsymbol{C}$, hippocampal lysates. Catenin subfamily levels are unchanged (A), as are nuclear $\beta$-catenin transcriptional target levels (B). C, Control animals (lanes 1-3) have comparable levels of activated Rac (top, precipitation with GST-PDB; bottom, lysate); conditional mutants (lanes $4-5$ ) have a significant decrease. D, Dact1 mutant hyperfilopodial phenotypes are rescued by RacCA but not by Cdc42CA, RhoCA, of dominant-negative Rac (RacDN), Cdc42 (Cdc42DN), or Rho (RhoDN). $\boldsymbol{E}$, Recombinant expression of Dact1 or RacCA rescues spine subtype distribution. ${ }^{*} p<0.05$, ${ }^{* *} p<0.01$; n.s., Not significant. All $p$ values in $\boldsymbol{D}$ and $\boldsymbol{E}$ are relative to wild type and when not indicated are not significant.

Wnt/ $\beta$-catenin signaling pathway, which controls target gene transcription by regulating nuclear $\beta$-catenin levels (Cheyette et al., 2002; Gloy et al., 2002; Jiang et al., 2008; Lagathu et al., 2009). Nonetheless, using quantitative RT-PCR, we could detect no significant or consistent differences between mutant and wild-type HCNs in basal levels of established neuronal $\beta$-catenin transcriptional target mRNAs, including Fos, Fosl, Axin1, and Axin2 (percentage of the wild-type average: for Fos, Dact $1^{+/+}, 100 \pm 13$ vs Dact $^{-/-}, 88 \pm 22, p=0.65$; for Fosl, Dact ${ }^{+/+}, 100 \pm 33$ vs Dact $1^{-/-}, 89 \pm 19, p=0.77$; for Axin 1, Dact $^{+/+}, 100 \pm 12$ vs Dact $^{-/-}, 114 \pm 15, p=0.47$; for Axin2, Dact ${ }^{+/+}, 100 \pm 28$ vs Dact $^{-/-}, 87 \pm 38, p=0.80$ ) (Fig. $4 B$ ).

Neurons and other cell types transfected with exogenous Dact1 undergo a dramatic alteration in cell morphology, forming aberrant lamellopodial and filopodial structures that stain strongly for actin (data not shown). This is reminiscent of actinbased morphological changes observed in cells that have undergone hyperactivation of the small GTPase Rac (Nakayama et al., 2000; Tashiro et al., 2000). Coexpression of dominant-negative mutant Rac blocks morphological changes in non-neuronal immortalized cells overexpressing Dact1 (data not shown), further suggesting that Dactl overexpression acts via Rac to produce these changes. Conversely, the observed increase in filopodia relative to mature spines in Dact1 ${ }^{-1}$ HCNs is consistent with reductions in Rac activity (Tashiro and Yuste, 2004). On this basis, we hypothesized that endogenous Dactl participates in activation of Rac and that Rac hypoactivation underlies spine maturation phenotypes in Dact1 ${ }^{-/-}$neurons. To test this hypothesis, we measured GTP-bound (activated) Rac normalized to total Rac in hippocampal lysates from adult mice in conditional mutant and control littermates. Lysates from conditional mutant hippocampi had significantly less activated Rac than control littermates (arbitrary units normalized to total Rac in control, $12.2 \pm$ 1.1 vs conditional mutant, $3.9 \pm 3.9$ arbitrary units normalized to total Rac, $p=0.027$ ) (Fig. 4C). We also examined mutant HCNs transfected with dominant-negative or constitutively active mutant forms of all three members of the Rho GTPase family (RhoA, Rac1, and Cdc42) and assessed the ability of these proteins to rescue Dact ${ }^{-1-}$ phenotypes, using Dact1-rescued HCNs as a positive control. Expression of either Dact1 or constitutively active Rac1 ( $\mathrm{RacCA}$ ) rescued the mutant hyper-filopodial phenotype, such that the proportion of filopodia was restored to wildtype levels in both these conditions $\left(\right.$ Dact $^{+/+}, 0.14 \pm 0.03$ vs Dact $^{-/-}, 0.35 \pm 0.05, p=0.0036 ;$ Dact $^{+/+}$vs Dact1-rescued, $0.24 \pm 0.03, p=0.11 ;$ Dact $^{+/+}$vs RacCA-rescued, $0.16 \pm 0.02$, $p=0.56$ ) (Fig. $4 D$ ). In contrast, expression of constitutively active versions of $\mathrm{Cdc} 42$ and RhoA or dominant-negative versions of Rac1, Cdc42, and RhoA did not rescue this phenotype (Fig. $4 D$ ). Impressively, expression of either Dactl or RacCA in mutant HCNs also normalized the distribution of mature spine types (thin spines, mushroom spines, and stubs), although a difference from wild type in the proportion of mushroom spines remained barely significant in mutant $\mathrm{HCNs}$ transfected with RacCA $\left(\right.$ Dact $^{+/+}, 0.54 \pm 0.05$ vs RacCA-rescued, $0.39 \pm 0.04, p=$ 0.047) (Fig. 4E). Together, our analyses indicate that the neural differentiation phenotypes we have characterized in Dact1 mutants are mediated substantially, if not exclusively, via downstream reductions in Rac activity.

\section{Discussion}

Loss of Dact1 in mammalian hippocampal neurons leads to decreases in dendrite complexity, excitatory synapse markers, and excitatory synaptic activity, without similar losses in inhibitory synapse markers. The effect of Dactl loss on excitatory synapses 
correlates with a loss of dendritic spines, which are the subcellular site specific to excitatory synapses in pyramidal neurons. This suggests that loss of Dact 1 impairs dendrite and spine formation and may have only secondary effects on excitatory synapse numbers. Nonetheless, we cannot rule out a separate requirement for Dact1 within excitatory synapses themselves (reflecting a role, for example, in adhesive and cytoskeletal interactions at the postsynaptic terminal), particularly because we have shown that Dactl is present at the synapse and because developmental relationships between spine and synapse formation are only beginning to be resolved (Kayser et al., 2008; Zito et al., 2009).

Our results suggest that a major function of Dact1 in differentiating neurons is to promote Rac-dependent actin rearrangements necessary for dendrite and spine formation. This might occur through regulation of levels of a catenin protein, such as p120-catenin, that in turn regulates Rac activity (Elia et al., 2006; Park et al., 2006). Against this, in Dact1 ${ }^{-/-}$HCNs, we have been unable to detect differences in levels of p120-catenin or $\delta$-catenin, nor in members of the $\alpha$-catenin and $\beta$-catenin subfamilies. It remains possible that loss of Dact 1 affects a small pool of signaling (e.g., soluble cytoplasmic or nuclear) catenin within neurons or more specifically in spine heads or excitatory synapses, but we have detected no differences in the subclass of catenin most clearly established to be involved in signaling ( $\beta$-catenin) nor in $\beta$-catenin transcriptional targets in neuronal lysates. Dact1 contains a PDZ-binding motif implicated in functions of synaptically localized adhesion molecules (Dalva et al., 2007), it binds to nuclear proteins that can alter gene activity through direct chromatin interactions (Hikasa and Sokol, 2004; Gao et al., 2008), and its non-catenin partners Dishevelled and Vangl2 have each been independently implicated in Rac regulation (Rosso et al., 2005; Lindqvist et al., 2010). This suggests that Dactl may be part of a dendrite, spine, and synapse differentiation pathway that includes transmembrane, cytoplasmic, and nuclear effectors. In future studies, we will identify other components of this emerging pathway and determine whether it is activated downstream of synaptically localized cell adhesion or secreted signaling molecules.

We have shown that Dact1 plays an important role during dendrite and spine formation in neurons of the mammalian forebrain by promoting activation of Rac. Other Dact family members are also expressed in neurons (Fisher et al., 2006) and are present at synapses (Trinidad et al., 2008) yet have been shown to bind and regulate distinct sets of signaling proteins ( $\mathrm{Su}$ et al., 2007). Through such divergent pathway functions, coexpressed Dact family members may play complementary nonredundant roles in creating and maintaining the highly polarized morphology and synaptic contacts of mammalian neurons.

\section{References}

Abe K, Takeichi M (2007) NMDA-receptor activation induces calpainmediated b-catenin cleavages for triggering gene expression. Neuron 53:387-397.

Brott BK, Sokol SY (2005) A vertebrate homolog of the cell cycle regulator Dbf4 is an inhibitor of Wnt signaling required for heart development. Dev Cell 8:703-715.

Cheyette BN, Waxman JS, Miller JR, Takemaru K, Sheldahl LC, Khlebtsova N, Fox EP, Earnest T, Moon RT (2002) Dapper, a Dishevelledassociated antagonist of beta-catenin and JNK signaling, is required for notochord formation. Dev Cell 2:449-461.

Cobos I, Borello U, Rubenstein JL (2007) Dlx transcription factors promote migration through repression of axon and dendrite growth. Neuron 54:873-888.
Dalva MB, McClelland AC, Kayser MS (2007) Cell adhesion molecules: signalling functions at the synapse. Nat Rev Neurosci 8:206-220.

Dao DY, Yang X, Chen D, Zuscik M, O’Keefe RJ (2007) Axin1 and Axin2 are regulated by TGF- $\beta$ and mediate cross-talk between TGF- $\beta$ and Wnt signaling pathways. Ann N Y Acad Sci 1116:82-99.

Elia LP, Yamamoto M, Zang K, Reichardt LF (2006) p120 catenin regulates dendritic spine and synapse development through Rho-family GTPases and cadherins. Neuron 51:43-56.

Fisher DA, Kivimäe S, Hoshino J, Suriben R, Martin PM, Baxter N, Cheyette BN (2006) Three Dact gene family members are expressed during embryonic development and in the adult brains of mice. Dev Dyn 235: 2620-2630.

Gao X, Wen J, Zhang L, Li X, Ning Y, Meng A, Chen YG (2008) Dapper1 is a nucleocytoplasmic shuttling protein that negatively modulates wnt signaling in the nucleus. J Biol Chem 283:35679-35688.

Garside ML, Turner PR, Austen B, Strehler EE, Beesley PW, Empson RM (2009) Molecular interactions of the plasma membrane calcium ATPase 2 at pre- and post-synaptic sites in rat cerebellum. Neuroscience 162: 383-395.

Gloy J, Hikasa H, Sokol SY (2002) Frodo interacts with Dishevelled to transduce Wnt signals. Nat Cell Biol 4:351-357.

Gorski JA, Talley T, Qiu M, Puelles L, Rubenstein JL, Jones KR (2002) Cortical excitatory neurons and glia, but not GABAergic neurons, are produced in the Emx1-expressing lineage. J Neurosci 22:6309-6314.

Habas R, He X (2006) Activation of Rho and Rac by Wnt/Frizzled signaling. Methods Enzymol 406:500-511.

Hell JW, Jahn R (1994) Preparation of synaptic vesicles from mammalian brain. In: Cell biology: a laboratory handbook (Celis JE, ed). New York: Academic.

Hering H, Sheng M (2001) Dendritic spines: structure, function and regulation. Nat Rev Neurosci 2:880-888.

Hikasa H, Sokol SY (2004) The involvement of Frodo in TCF-dependent signaling and neural tissue development. Development 131:4725-4734.

Jiang X, Tan J, Li J, Kivimäe S, Yang X, Zhuang L, Lee PL, Chan MT, Stanton LW, Liu ET, Cheyette BN, Yu Q (2008) Dact3 is an epigenetic regulator of Wnt/b-catenin signaling in colorectal cancer and is a therapeutic target of histone modifications. Cancer Cell 13:529-541.

Kayser MS, Nolt MJ, Dalva MB (2008) EphB receptors couple dendritic filopodia motility to synapse formation. Neuron 59:56-69.

Lagathu C, Christodoulides C, Virtue S, Cawthorn WP, Franzin C, Kimber WA, Nora ED, Campbell M, Medina-Gomez G, Cheyette BN, Vidal-Puig AJ, Sethi JK (2009) Dact1, a nutritionally regulated preadipocyte gene, controls apidogenesis by coordinating the Wnt/b-catenin signaling network. Diabetes 58:609-619.

Lee SH, Peng IF, Ng YG, Yanagisawa M, Bamji SX, Elia LP, Balsamo J, Lilien J, Anastasiadis PZ, Ullian EM, Reichardt LF (2008) Synapses are regulated by the cytoplasmic tyrosine kinase Fer in a pathway mediated by p120 catenin, Fer, Shp-2, and b-catenin. J Cell Biol 183:893-908.

Lindqvist M, Horn Z, Bryja V, Schulte G, Papachristou P, Ajima R, Dyberg C, Arenas E, Yamaguchi TP, Lagercrantz H, Ringstedt T (2010) Vang-like protein 2 and Racl interact to regulate adherens junctions. J Cell Sci 123:472-483.

Long JE, Cobos I, Potter GB, Rubenstein JL (2009) Dlx1\&2 and Mash1 transcription factors control MGE and CGE patterning and differentiation through parallel and overlapping pathways. Cereb Cortex 19 [Suppl 1]:i96-i106.

Nakayama AY, Harms MB, Luo L (2000) Small GTPases Rac and Rho in the maintenance of dendritic spines and branches in hippocampal pyramidal neurons. J Neurosci 20:5329-5338.

Niwa H, Yamamura K, Miyazaki J (1991) Efficient selection for high-expression transfectants with a novel eukaryotic vector. Gene 108:193-199.

Park JI, Ji H, Jun S, Gu D, Hikasa H, Li L, Sokol SY, McCrea PD (2006) Frodo links Dishevelled to the p120-catenin/Kaiso pathway: distinct catenin subfamilies promote Wnt signals. Dev Cell 11:683-695.

Rosso SB, Sussman D, Wynshaw-Boris A, Salinas PC (2005) Wnt signalling through Dishevelled, Rac and Jnk regulates dendritic development. Nat Neurosci 8:34-42.

Rothe C, Urlinger S, Löhning C, Prassler J, Stark Y, Jäger U, Hubner B, Bardroff M, Pradel I, Boss M, Bittlingmaier R, Bataa T, Frisch C, Brocks B, Honegger A, Urban M (2008) The human combinatorial antibody library HuCAL GOLD combines diversification of all six CDRs according to the natural immune system with a novel display method for efficient selection of high-affinity antibodies. J Mol Biol 376:1182-1200. 
Sala C, Cambianica I, Rosso F (2008) Molecular mechanisms of dendritic spine development and maintenance. Acta Neurobiol Exp (Wars) 68:289-304.

Su Y, Zhang L, Gao X, Meng F, Wen J, Zhou H, Meng A, Chen YG (2007) The evolutionally conserved activity of Dapper2 in antagonizing TGFbeta signaling. FASEB J 21:682-690.

Sun T, Patoine C, Abu-Khalil A, Visvader J, Sum E, Cherry TJ, Orkin SH, Geschwind DH, Walsh CA (2005) Early asymmetry of gene transcription in embryonic human left and right cerebral cortex. Science 308:1794-1798.

Suriben R, Kivimäe S, Fisher DA, Moon RT, Cheyette BN (2009) Posterior malformations in Dactl mutant mice arise through misregulated Vangl2 at the primitive streak. Nat Genet 41:977-985.
Tashiro A, Yuste R (2004) Regulation of dendritic spine mobility and stability by Racl and Rho kinase: evidence for two forms of spine motility. Mo Cell Neurosci 26:429-440.

Tashiro A, Minden A, Yuste R (2000) Regulation of dendritic spine morphology by the Rho family of small GTPases: antagonistic roles of Rac and Rho. Cereb Cortex 10:927-938.

Trinidad JC, Thalhammer A, Specht CG, Lynn AJ, Baker PR, Schoepfer R Burlingame AL (2008) Quantitative analysis of synaptic phosphorylation and protein expression. Mol Cell Proteomics 7:684-696.

Urbanska M, Blazejczyk M, Jaworski J (2008) Molecular basis of dendritic development. Acta Neurobiol Exp (Wars) 68:264-288.

Zito K, Scheuss V, Knott G, Hill T, Svoboda K (2009) Rapid functional maturation of nascent dendritic spines. Neuron 61:247-258. 